\title{
Update on coaxial coupling scheme for International Linear Collider-type cavities
}

\author{
P. Kneisel ${ }^{1, *}$ and J. Sekutowicz ${ }^{2}$ \\ ${ }^{1}$ Thomas Jefferson National Accelerator Facility, Newport News, Virginia 23606, USA \\ ${ }^{2}$ Deutsches Elektronen Synchrotron DESY, Notkestrasse 85, 22603 Hamburg, Germany \\ (Received 30 November 2009; published 17 February 2010)
}

\begin{abstract}
This paper reports on our efforts to develop a flangeable coaxial coupler for both higher order mode and fundamental coupling for nine-cell ILC-type cavities, which were designed in the early 1990's for pulsed operation with a duty factor less than $1 \%$. The design of the coupler has been done in such a way that the rf magnetic flux $B$ at the flange connection was minimized and only a field of $<5 \mathrm{mT}$ would be present for an operation at an accelerating field $E_{\text {acc }} \sim 36 \mathrm{MV} / \mathrm{m}(B \sim 150 \mathrm{mT})$ in the cavity. Even though we achieved reasonably high $Q$ values at low field, the cavity/coupler combination was limited in the $\mathrm{cw}$ mode to only $\sim 7 \mathrm{MV} / \mathrm{m}$, where a thermally initiated degradation occurred. We believed that this limitation was caused by poor cooling of the shorting plate and inner tube in the coaxial coupler; therefore, we have improved the cooling conditions by initially drilling radial cooling channels every 30 degrees, then every 15 degrees into the shorting plate and eventually removing the "bridges" between the channels. This paper reports on our experiences with the modified coaxial coupler under cw and pulsed conditions.
\end{abstract}

DOI: 10.1103/PhysRevSTAB.13.022001

PACS numbers: $74.90 .+n$

\section{INTRODUCTION}

In the coaxial coupling (CC) scheme, shown in Fig. 1, both fundamental power coupler (FPC) and higher order mode (HOM) couplers are shielded by the inner tube, which is supported by the $\mathrm{Nb}$ disk welded to it and to the beam tube. The disk is an electric short in the coaxial line, which is formed by the inner and outer tubes, and thus separates electrically two mirrored coupling devices and neighboring cavities. The pair of mirrored coupling devices can be flanged between two cavities. The flanges are located $\sim 35 \mathrm{~mm}$ apart from the end irises, at the positions where the standing wave of the magnetic flux has its notch.

The coaxial HOM couplers were originally developed for the $500 \mathrm{MHz}$ HERA cavities in 1985 and later in the early 1990's they were scaled to $1300 \mathrm{MHz}$ and adapted for the TESLA cavities [1]. The TESLA HOM damping scheme fulfills also the specification for the ILC project, which is the TESLA successor, and can be used for the superconducting cavities in the main accelerator.

\section{Motivation}

This coaxial coupling scheme has the following advantages as compared to the standard TESLA/ILC scheme: (i) Field asymmetries and kicks from all couplers are minimized. (ii) The distance between two cavities can be shorter (higher real estate gradient); for the ILC, the difference is $9 \mathrm{~cm}$. (iii) The body of the cavity stays cylindrically symmetric, which enables its fabrication by hydroforming as a seamless device. (iv) The interior of

\footnotetext{
*kneisel@jlab.org
}

the coupling assembly and the cavities can be better cleaned before the final assembly.

\section{MODELING AND BENCH RF MEASUREMENTS}

(i) HOM damping and coupling to the fundamental mode have been modeled by the advanced computation department team at SLAC. For the accelerating mode, the computed $Q_{\text {ext }}$ for the FPC covered a wide range of values beginning at a lowest value of $10^{5}$ depending on the penetration of the coupler probe into the coaxial line. For the first two HOM passbands, the calculated damping compares favorably with the TESLA Technical Design Report damping scheme [1].

(ii) The second step in the verification of the concept was the HOM damping and FM coupling measurements with a copper model of the coupling device attached to the copper model of the TESLA cavity. The models have been built and tested at JLab. The agreement between calculated and
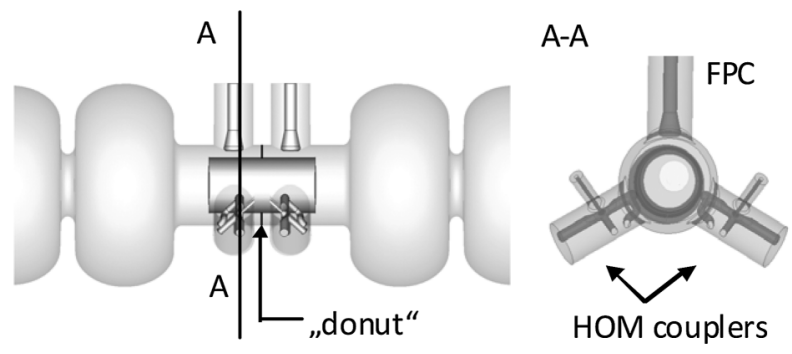

FIG. 1. FPC and HOM couplers in two mirrored coaxial coupling devices placed between two cavities (left) and cross section of the coupling device (right). 


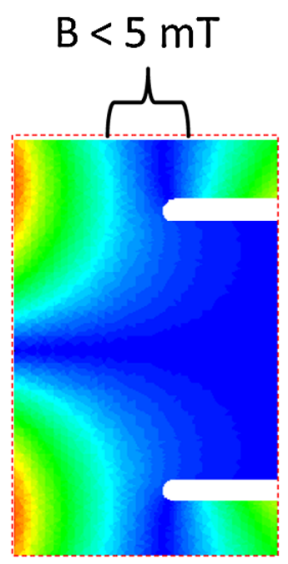

FIG. 2. (Color) $B$-field pattern in the beam tube at the joint.

measured HOM damping of the first two passbands was remarkable; more information can be found in [1]; for three dipole passbands $\left(\mathrm{TE}_{111}, \mathrm{TM}_{110}\right.$, and $\left.\mathrm{TE}_{121}\right)$ and for the lowest monopole passband $\left(\mathrm{TM}_{011}\right)$ the damping specification for the ILC $-Q_{\text {ext }}<2 \times 10^{5}$-is fulfilled.

(iii) Fundamental power coupler $Q_{\text {ext }}$ measurements as a function of coax-line penetration into the beam pipe have been done at DESY and an optimum geometrical configuration has been determined for minimizing the magnetic field at the location of the superconducting flange connection. The reflected wave at the $\mathrm{Nb}$ short in the coaxial line forms with the decaying field in the beam tube a standing wave pattern, having a $B$-field minimum ("notch") at $45 \mathrm{~mm}$ from the end iris. A superconducting connection made of a $\mathrm{Nb}$ gasket and $\mathrm{NbZr}$ flanges at that location will be exposed to a negligible residual magnetic flux. Figure 2 shows the field pattern and field amplitude for an accelerating gradient of $E_{\text {acc }}=36 \mathrm{MV} / \mathrm{m}(B$-field $=150 \mathrm{mT})$ in the cavity.

More information about the modeling and bench measurements can be found in [1].

\section{PREPARATION FOR TESTING}

The $\mathrm{Nb}$ prototype of the $\mathrm{CC}$ device was built at TJNAF in 2008. The prototype is simplified compared to Fig. 1 because it is equipped with only two HOM couplers and has no input coupler port.

We chose a 1.6-cell superconducting radio frequency (SRF) gun cavity, built in the frame of another superconducting R\&D project, for the cold tests of the CC device. The cavity and CC device are shown in Fig. 3.

Both the $\mathrm{CC}$ device and the cavity have electron beam welded conflat (CF) flanges, made of the $\mathrm{Nb} 1 \% \mathrm{Zr}$ alloy, for the superconducting connection. The cavity itself is made of large grain high residual resistivity ratio niobium. We chose NbZr as flange material because of its good mechanical stability and its better superconducting properties in comparison to $\mathrm{Nb} 55 \% \mathrm{Ti}$ as experimentally verified in Ref. [2]. However, the mechanical properties of the material we used for the CF flanges turned out to be too soft and the CF knife edge deformed when used with an annealed niobium gasket. Therefore, after each assembly the knife edge had to be "resharpened"; this procedure seemed to work reasonably well, since the niobium gasket is also the vacuum seal and the cavity assembly was leak tight at cryogenic temperatures. On the other hand, as can be seen below, we experienced some variation in the low $E_{\text {acc }} Q$ value, which we tend to attribute to variations in the knife edge/niobium gasket topography, resulting in a more or less lossy contact. Figure 4 shows a close-up picture of the $\mathrm{Nb} 1 \% \mathrm{Zr}$ conflat flange and the niobium gasket after disassembly.

\section{CRYOGENIC TESTS}

Here we report only about tests subsequent to the ones reported in Ref. [3].

We have carried out four additional experiments since PAC 2009; all four employed single crystal, annealed
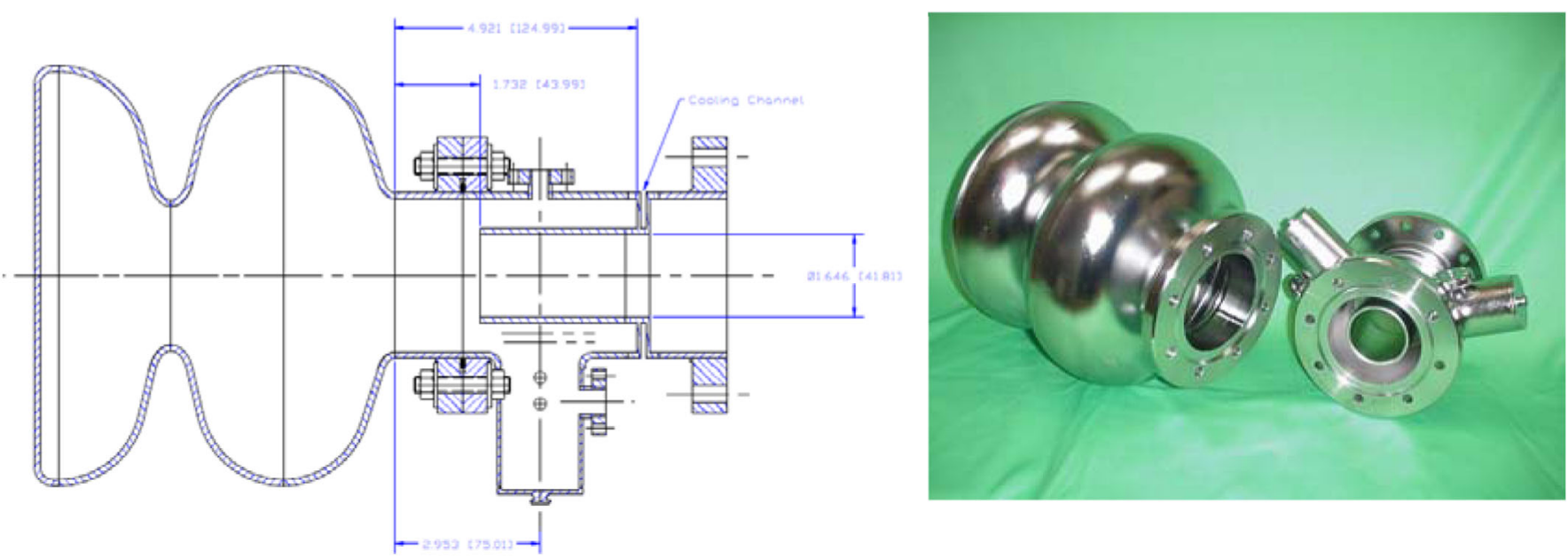

FIG. 3. (Color) 1.6-cell SRF gun cavity and coaxial coupling device: assembly drawing and parts before assembly. 


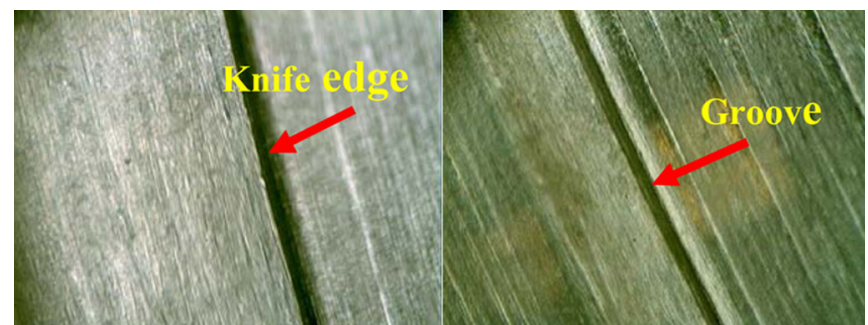

FIG. 4. (Color) Close-up pictures of NbZr knife edge and niobium gasket groove. Both surfaces are less than ideal.

niobium gaskets. For tests No. 2 and No. 3, cooling channels had been drilled radially into the shorting plate between the inner and outer conductor every 30 degrees and 15 degrees, respectively, as can be seen in Fig. 5, whereas test No. 1 was carried out without cooling channels, and in test No. 4 the "bridges" between the channels were completely removed.

Prior to each assembly, the knife edges on the cavity and the coaxial coupler assembly were mechanically "touched up" with a polishing stone usually used for deburring of lathe cutting tools. Both the conflat flanges and the mechanically polished niobium gaskets received a slight buffered chemical polishing treatment prior to assembly. Cavity and coaxial coupler were high pressure rinse and subsequently dried in our class 10 clean room prior to assembly.

In all tests we encountered some multipacting (MP) at low fields, which could be overcome by rf processing. The most severe MP occurred in test No. 2 and it took several hours to pass through the MP level. However, after disassembly of the cavity we found some residue from masking tape; this contamination was accidentally overlooked

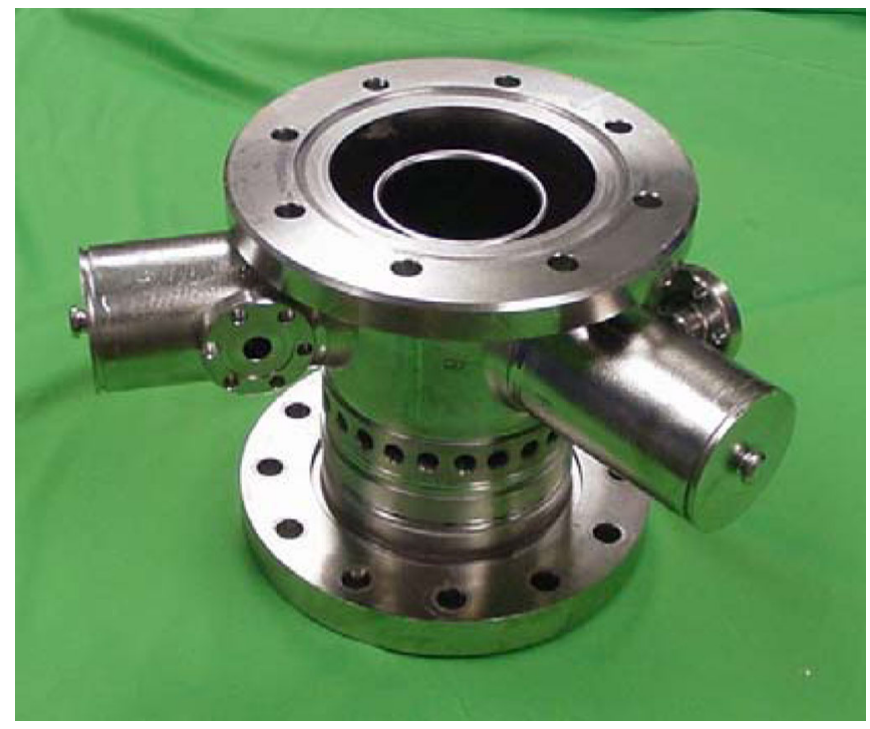

FIG. 5. (Color) Modified coaxial coupler assembly with cooling channels every 15 degrees.

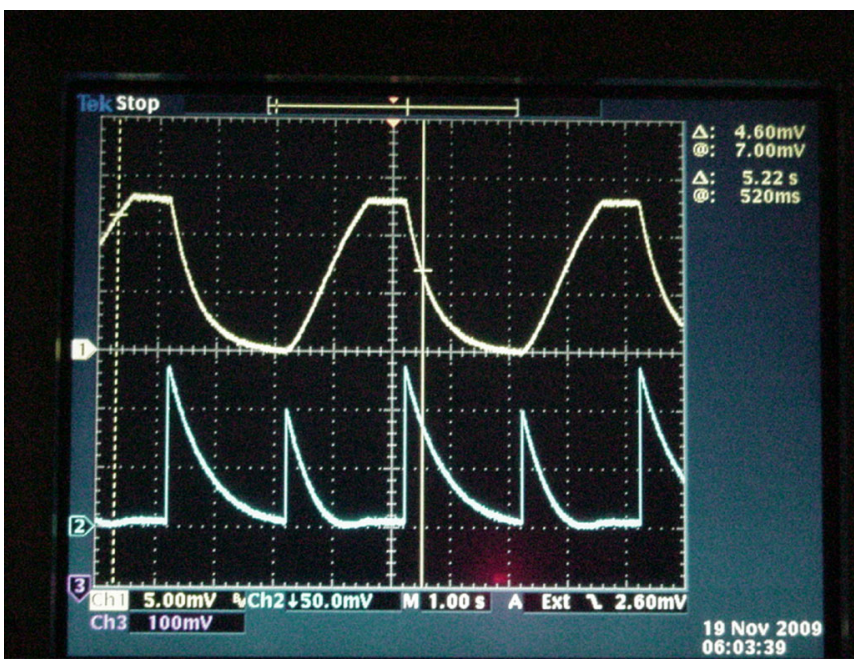

FIG. 6. (Color) Multipacting barrier encountered in the coaxial line: upper trace, transmitted signal; lower trace, reflected signal.

during cavity preparation and assembly. Usually the MP processed in less than $30 \mathrm{~min}$.

A typical trace of a multipacting barrier is shown in Fig. 6: the cavity is operated under pulsed conditions, the upper trace shows the transmitted signal and the lower trace is the reflected signal. The "flat top" is an indication of the barrier.

The test results are summarized in Fig. 7, indicating that the cooling of the inner conductor is a problem in the original design and could be improved marginally by drilling radial cooling channels into the shorting plate between inner and outer conductor. However, no further improvement was realized in test No. 4 after the removal of the bridges between the holes. As a matter of fact, in this latest test heating occurred already at lower fields than in the previous tests and a gradient of $\sim 8 \mathrm{MV} / \mathrm{m}$ could only be achieved by pulsing the input with a duty factor of $50 \%$ power. This is somewhat disappointing and points out the need for a better design of the system as discussed later. In

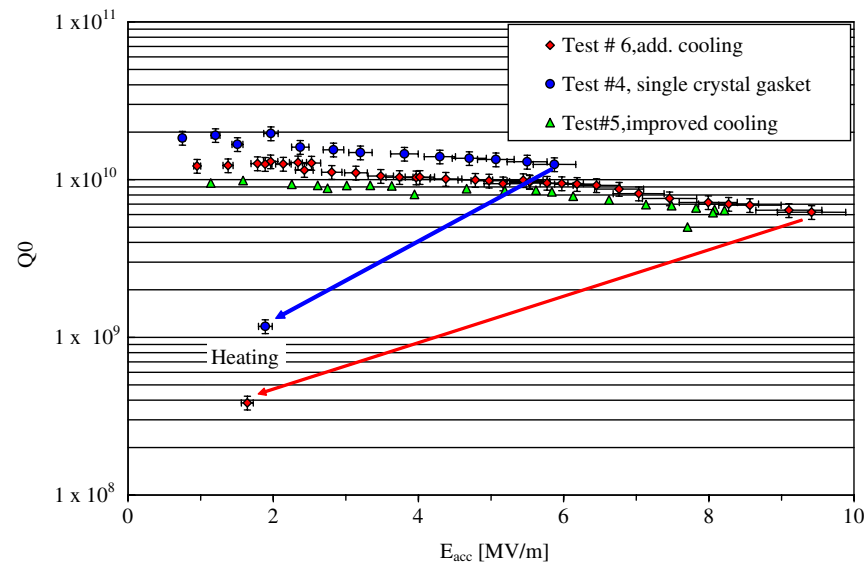

FIG. 7. (Color) Results from tests with single crystal gaskets and improved cooling. 
this particular test the vacuum performance of the cavity was the best in all tests-in superfluid helium the cavity held a vacuum of $p<4 \times 10^{-9}$ mbar over several daysbut also shows that vacuum integrity of a connection and good sc contact are two different things.

\section{SUMMARY AND OUTLOOK}

Even though the concept of a flange-on connection of a coaxial coupling assembly onto a multicell cavity is rather straightforward and computer modeling as well as room temperature developments and tests provided excellent agreements, the practical implementation into a superconducting niobium assembly is not as easy.

Our present experience/design confronted us with two problems: (i) The edges of the Nb1\%Zr flanges are strongly deformed after each assembly and the material hardness of the material we used was below the expected value reported by the vendor $(20 \%$ below the Vickers hardness of 125). We subsequently learned from the material supplier that the mechanical strength of the material strongly depends on the annealing conditions during material casting and rolling and can be improved. (ii) The original design of the coaxial coupler components did not provide sufficient cooling to the shorting plate between inner and outer conductors. Some marginal improvements were achieved with radial cooling channels, but these are not sufficient for the cw and high duty factor pulse operation at high gradients.

The way forward to better performance of the assembly will be a replacement of the present $\mathrm{Nb} 1 \% \mathrm{Zr}$ flanges with material of higher hardness, which will better withstand the pressures applied during the assembly with the gasket. If no deformation occurs, a rework with a grinding stone as mentioned above would not be necessary and it should be possible to achieve a much better surface finish (see Fig. 4) on the knife edges during initial careful machining-this also applies to the $\mathrm{Nb}$ gaskets. In this case we expect a better reproducibility of the high $Q$ values-we attribute the present variation in $Q$ value to variations in the surface finish of knife edges and gaskets.

The second modification needs to be an improvement of the cooling conditions. We hope to be able to construct the inner conductor as a double wall tube for better heat transfer.

We plan to implement these modifications in the near future.

Finally, in the future we will conduct low $(\sim 1 \%)$ duty factor tests in addition to the standard $\mathrm{cw}$ tests to investigate the CC device under conditions closer to the ILC operation mode.

\section{ACKNOWLEDGMENTS}

We would like to thank our colleagues G. Slack, L. Turlington, and P. Kushnik from TJNAF for their support of this work. This manuscript has been authored by Jefferson Science Associates, LLC under U.S. DOE Contract No. DE-AC05-06OR23177.

[1] J. Sekutowicz and P. Kneisel, Proceedings of the 2008 Linear Accelerator Conference, Victoria, Canada, 2008, paper THP044, http://accelconf.web.cern.ch/accelconf/.

[2] P. Kneisel et al., Applied Superconductivity Conference 2008, Chicago, 2008, paper 3LPB02.

[3] J. Sekutowicz and P. Kneisel, Proceedings of the 2009 Particle Accelerator Conference, Vancouver, Canada, 2009, paper TU5PFP05, http://accelconf.web.cern.ch/ accelconf/. 